\title{
Compact high-power terahertz radiation source
}

\author{
G. A. Krafft \\ Center for Advanced Studies of Accelerators, Jefferson Laboratory, Newport News, Virginia 23608, USA
}

(Received 11 September 2003; published 21 June 2004)

\begin{abstract}
In this paper a new type of $\mathrm{THz}$ radiation source, based on recirculating an electron beam through a high gradient superconducting radio frequency cavity, and using this beam to drive a standard electromagnetic undulator on the return leg, is discussed. Because the beam is recirculated and not stored, short bunches may be produced that radiate coherently in the undulator, yielding exceptionally high average $\mathrm{THz}$ power for relatively low average beam power. Deceleration from the coherent emission, and the detuning it causes, limits the charge-per-bunch possible in such a device.
\end{abstract}

DOI: 10.1103/PhysRevSTAB.7.060704

PACS numbers: 41.75.Lx, 29.27.Bd, 41.60.Cr

In the past two years, there have been a number of papers published addressing the subject of producing $\mathrm{THz}$ radiation through the coherent synchrotron radiation (CSR) emission process [1-3]. These studies assume a storage ring source; the CSR is produced by coherent synchrotron emission from density variations at wavelengths short compared to the overall bunch length in the storage ring. Initial work focused on measuring, quantifying, and theoretically explaining $[3,4]$ the early observations of transient bursting in this emission [5]. A recent work claims a steady $\mathrm{THz}$ source in a storage ring has been observed [6]. The primary purpose of this paper is to point out that recirculated linac-based sources of coherent $\mathrm{THz}$ radiation offer the promise of higher power compared to ring-based sources, primarily because the electron pulse lengths possible from recirculated linacs may be made shorter than the emission wavelength, whereas the emission in storage rings is limited by the density modulation possible. To illustrate the advantages of the recirculated linac approach, some ideas regarding compact $\mathrm{THz}$ sources are presented by a high level design of such a source.

Coherent synchrotron radiation [7], and more recently coherent transition radiation [8] and coherent undulator radiation (CUR) [9], have been used for a number of years for electron beam diagnostic purposes [10,11]. In a typical application one has a short bunch emerging from a linear accelerator, and the electromagnetic radiation emitted from the bunch by a bending magnet, a transition radiation foil, or an undulator, is measured and frequency analyzed. In the small source approximation the energy per unit frequency per unit solid angle emitted by a single passage of an electron bunch with $N_{e}$ electrons through a radiator is

$$
\frac{d^{2} E}{d \omega d \Omega}(\omega)=\left.N_{e} \frac{d^{2} E}{d \omega d \Omega}\right|_{1 e}\left(1+N_{e}|S(\omega)|^{2}\right),
$$

where $\left.\frac{d^{2} E}{d \omega d \Omega}\right|_{1 e}$ is the energy per unit angular frequency per unit solid angle emitted by a single electron, and $S(\omega)$ is the Fourier transform of the unit normalized bunch longitudinal distribution $I(z)$,

$$
S(\omega)=\int I(z) e^{-i \omega z / c} d z, \quad \int I(z) d z=1 .
$$

Coherent emission occurs at those wavelengths at which the form factor, $|S(\omega)|^{2}$, is of the order of 1 ; at these wavelengths, typically longer than the bunch length, the energy goes as $N_{e}^{2}$, as does the power emitted by a continuous repetitive stream of such bunches. In the beam diagnostic applications, one usually concentrates on wavelengths comparable to the bunch length or larger, because it is at these wavelengths that the transition between coherent and incoherent emission occurs. In the fully coherent limit, notice that the total power emitted goes as the charge-per-bunch squared, and to get maximum emission it is advantageous to obtain as short a bunch as possible.

Recirculated linacs, the largest example of which is Jefferson Lab's Continuous Electron Beam Accelerator Facility (CEBAF) [12], have a number of desirable features that may make them interesting light sources $[13,14]$. For example, such accelerators may have superior emittance than is possible in storage rings, and they may produce and accelerate to high energy short electron pulses [15], which may be used to produce short electromagnetic radiation pulses, much shorter than is typical in storage rings. It is sufficient to note that electron pulse lengths of under $100 \mathrm{fs}(30 \mu \mathrm{m}) \mathrm{rms}$ have been observed at CEBAF with low bunch charge under $1 \mathrm{pC}$ [16], and $360 \mathrm{fs}(110 \mu \mathrm{m}) \mathrm{rms}$ has been observed on the Jefferson Lab infrared demonstration free electron laser (IR DEMO FEL) [17] at $60 \mathrm{pC}$ bunch charge. These short longitudinal dimensions have been observed by a variety of techniques, including analysis of the spectrum of coherent synchrotron radiation and coherent transition radiation, as discussed above.

It is difficult to find high average power sources of coherent electromagnetic radiation for wavelengths between $0.1-1 \mathrm{~mm}(3-0.3 \mathrm{THz})$. This wavelength regime, sometimes referred to as the $\mathrm{THz}$ gap, is beyond the reach of typical microwave production techniques, and also in a 


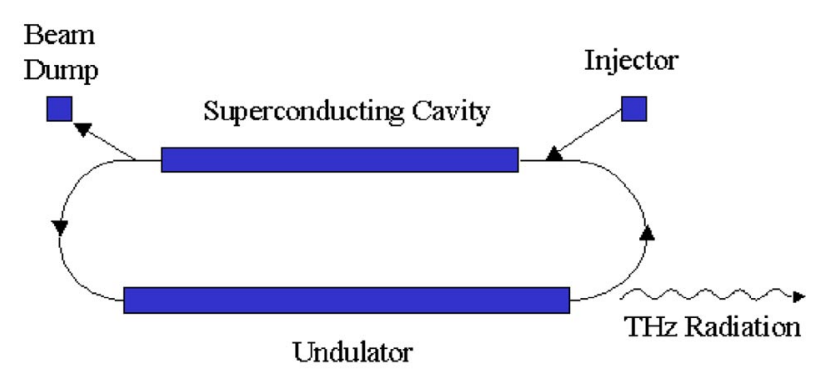

FIG. 1. (Color) Compact high average power THz source. The undulator is $1.25 \mathrm{~m}$ long in the case considered.

wavelength regime that is long enough that the strong transitions needed to construct a conventional laser are rare and difficult to use. Because the bunch length may be made smaller in a recirculated linac than the desired minimum wavelength, and because $\mathrm{THz}$ brilliance will be maximized by utilizing undulator emission, a device roughly as in Fig. 1 makes an interesting $\mathrm{THz}$ source. Some beam parameters are listed in Table I. A $300 \mathrm{keV}$ beam of electrons of $100 \mu \mathrm{A}$ average current originates from a photocathode gun. The beam is bunched with a single buncher cavity in the injector, and merged on the accelerator axis. In order to continue the bunching, the electron beam is accelerated slightly off crest. The first turnaround "arc" is chosen to have the correct $M_{56}$ to yield maximum bunching at the undulator. After emitting coherent undulator radiation, the beam is directed back through the superconducting rf cavity on a decelerating phase. The beam is dumped at low energy and low beam power.

None of the parameters in Table I stretch the state-ofthe-art terribly. For example, a single CEBAF $1497 \mathrm{MHz}$ 7-cell superconducting cavity of length $70 \mathrm{~cm}$ will yield about $12.6 \mathrm{MV}$ when operated at $18 \mathrm{MV} / \mathrm{m}$. Such a gradient performance is below the requirements for the planned upgrade for CEBAF, and covers the full operating energy range from $0.3-3 \mathrm{THz}$ as shown in Table II . The CEBAF accelerator routinely accelerates average currents of the order of $100 \mu \mathrm{A}$, and the Jefferson Lab IR FEL $5 \mathrm{~mA}$. The most interesting parameter choice is the charge per beam bunch. Assuming an untapered undulator, because one would like the same undulator to

TABLE I. THz source accelerator parameters.

\begin{tabular}{lcc}
\hline \hline \multicolumn{1}{c}{ Quantity } & Value & Unit \\
\hline Beam energy & $3.1-9.9$ & $\mathrm{MeV}$ \\
Average beam current & 100 & $\mu \mathrm{A}$ \\
Charge per beam bunch & 12 & $\mathrm{pC}$ \\
Bunch repetition rate & 8.3 & $\mathrm{MHz}$ \\
Normalized rms beam emittance & 5 & $\mathrm{~mm} \mathrm{mrad}$ \\
Longitudinal rms emittance & 10 & $\mathrm{keV} \mathrm{degrees}$ \\
rms bunch length at wiggler & $300(90)$ & $\mathrm{fsec}(\mu \mathrm{m})$ \\
\hline \hline
\end{tabular}

cover a wide range of operating conditions, the charge per bunch is limited by the fact that beam deceleration from the coherent emission should not cause substantial detuning of the emission. If the beam is fully bunched longitudinally, the total energy of the coherent undulator emission, $E_{\mathrm{CUR}}$, is approximately

$$
E_{\mathrm{CUR}}=N_{e}^{2} \frac{2 \pi}{6} \alpha N K^{2} h \frac{c}{\lambda}=\frac{4 \pi^{2}}{6} \frac{N_{e}^{2} e^{2}}{\lambda} N K^{2},
$$

where $N_{e}$ is the number of electrons in the bunch, $\alpha$ is the fine-structure constant, $N$ is the number of undulator periods, $K$ is the field strength parameter, $\lambda$ is the emission wavelength, $h$ is Planck's constant, and $c$ is the velocity of light. The emission wavelength is related to the undulator period $\lambda_{0}$ by the usual free electron laser resonance condition

$$
\lambda=\frac{\lambda_{0}}{2 \gamma^{2}}\left(1+K^{2} / 2\right) .
$$

The electron bunch energy is $E_{\text {beam }}=N_{e} \gamma m c^{2}$, and by requiring, conservatively, that $E_{\mathrm{CUR}} / E_{\text {beam }}<1 / 4 N$, a bunch charge limit of

$$
N_{e}<\frac{\gamma \lambda}{r_{e}} \frac{3}{8 \pi^{2} N^{2} K^{2}}
$$

is obtained where $r_{e}$ is the classical electron radius. For $1 \mathrm{THz}$ emission from a $5.7 \mathrm{MeV}$ beam driving a 25 period undulator, the maximum bunch charge is about $12 \mathrm{pC}$, and for a 3 period undulator the maximum bunch charge is almost a nanoCoulomb. This limit is not a hard limit because tapering the undulator may yield a higher possible bunch charge in a "single-frequency" $\mathrm{THz}$ source design. However, the estimate is instructive in that it provides some indication of the maximum output from a general-purpose $\mathrm{THz}$ source, which is obtained by filling every possible accelerating phase in the accelerator with this maximum charge. In this case an estimate of the "maximum" power possible in this type of device is obtained,

$$
P_{\mathrm{CUR}}<f_{\mathrm{rf}} \frac{3 \alpha\left(1+K^{2} / 2\right)}{128 \pi^{3} N^{3} K^{2}} h \frac{\lambda_{0} c}{r_{e}^{2}},
$$

where $f_{\text {rf }}$ is the rf frequency of the accelerator. From a total power viewpoint it is advantageous to increase the bunch repetition rate, increase the undulator period, and decrease the number of periods and the field strength because this increases the charge per bunch possible.

Assuming the same beam properties and the same average current, the total $\mathrm{THz}$ power from storage rings is not as large. First, for energies typical in present day storage rings, undulators tuned to the $\mathrm{THz}$ band would have to have unrealistically long period length, and one is forced to consider sources based on coherent synchrotron radiation from the bending magnets in the ring. Next, by assuming that the beam has a density modulation at 
TABLE II. THz source undulator and calculated optical parameters.

\begin{tabular}{|c|c|c|}
\hline Quantity & Value & Unit \\
\hline \multicolumn{3}{|l|}{ Undulator } \\
\hline Period length & 5 & $\mathrm{~cm}$ \\
\hline Period number & 3,25 & \\
\hline Field strength, $K=e B \lambda_{0} / 2 \pi m c^{2}$ & 1 & \\
\hline Wavelength at $5.7 \mathrm{MeV}$ & 0.3 & $\mathrm{~mm}$ \\
\hline Fundamental optical power & $0.7,5.9$ & $\mathrm{~W}$ \\
\hline Fundamental flux & $0.9 \times 10^{18}, 7.3 \times 10^{18}$ & photon/s in $0.1 \%$ bandwidth \\
\hline Fundamental brilliance & $1.6 \times 10^{13}, 2.8 \times 10^{14}$ & photon $/\left(\mathrm{s} \mathrm{mm}^{2} \mathrm{mrad}^{2}\right)$ in $0.1 \%$ bandwidth \\
\hline Optical pulse length $(N \lambda)$ & $0.9,7.5$ & $\mathrm{~mm}$ \\
\hline
\end{tabular}

wavelength $\lambda$ inside a Gaussian distribution with longitudinal extent $\sigma$,

$$
\begin{aligned}
I(z)= & \frac{1-m}{\sqrt{2 \pi} \sigma} \exp \left(-z^{2} / 2 \sigma^{2}\right) \\
+ & \frac{m}{\sqrt{2 \pi} \sigma} \frac{1}{1-\exp \left(-2 \pi^{2} \sigma^{2} / \lambda^{2}\right)} \\
& \times \exp \left(-z^{2} / 2 \sigma^{2}\right)[1+\cos (2 \pi z / \lambda)], \quad 0<m<1,
\end{aligned}
$$

where $m$ is the fractional longitudinal modulation, the total power radiated into each harmonic by coherent synchrotron radiation in free space is [18]

$$
P_{n} \approx 0.52 \frac{N_{e}^{2} e^{2} c}{\rho^{2}} n^{1 / 3}\left|S\left(n \omega_{0}\right)\right|^{2},
$$

where $n$ is the harmonic number, $\rho$ is the bend radius, and $\omega_{0}=c / \rho$ is the fundamental angular frequency of the revolution. Summing the form factor around $n_{0}=$ $2 \pi \rho / \lambda$, and using the fact that the weak $n^{1 / 3}$ dependence of the spectrum does not change much during the sum, the total energy-per-turn radiated at wavelengths close to the modulation wavelength is

$$
E_{\mathrm{CSR}} \approx 3.25 \frac{N_{e}^{2} e^{2}}{\rho} n_{0}^{1 / 3} \frac{\sqrt{\pi} \rho}{\sigma} \frac{m^{2}}{4} \ll E_{\mathrm{CUR}} .
$$

Because this energy is radiated over the whole ring circumference, and because at longer wavelengths the emission is suppressed due to shielding effects, it is clear that such a source is not as powerful as the undulator-based source.

In Table I, the repetition rate has been chosen to provide a convenient and demonstrated average current to work with, $100 \mu \mathrm{A}$, consistent with $12 \mathrm{pC}$ derived above. It should be borne in mind that an additional factor of 200 in average power is possible if a $20 \mathrm{~mA}$ injector is developed to fill every accelerating phase with $12 \mathrm{pC}$ of bunch charge. Strictly speaking, beam recirculation and beam energy recovery are essential source design elements only in a device at high average current.

The beam quality figures in Table I are estimates based on measurements performed on the IR DEMO FEL at Jefferson Lab [17]. As can be demonstrated by a simple calculation, $10 \mathrm{keV}$ degrees longitudinal emittance is consistent with $\sigma_{E} / E \leq 1 / 4 N=1 \%$ and a bunch length of $90 \mu \mathrm{m}$. To manipulate the longitudinal phase space in the way needed requires a buncher with a $\mathrm{cw}$ accelerating voltage of about $200 \mathrm{kV}$, a merging region $M_{56}$ of $-13 \mathrm{~cm}$ (the same in Ref. [17], but at much lower energy), and accelerating off crest by $3.3^{\circ}$ through the turnaround arc which has a bend radius of $20 \mathrm{~cm}$ and $M_{56}$ of $63 \mathrm{~cm}$. The dispersion at the undulator is $40 \mathrm{~cm}$, a value that causes inconsequential growth of the spot size at the undulator.

Consistent with Table I, some undulator parameters and photon beam characteristics are given in Table II for two cases of interest: first a few transverse oscillation period undulator which will yield a relatively wideband source, and a 25 period undulator which yields a relatively narrow band source. Undulators with such parameters are by now entirely standard and well within the state-of-theart. The average fundamental optical power is estimated using Eq. (1). The fundamental flux into a bandwidth $\Delta \omega / \omega$ is estimated as

$$
F=f N_{e}^{2} \frac{\pi}{2} \alpha N \frac{\Delta \omega}{\omega}[J J]
$$

where $[J J]$ is a standard Bessel function factor for which $K=1$ is around 0.55 for fundamental emission.

A general estimate for the brilliance is [19]

$$
B=\frac{F}{(2 \pi)^{2} \sqrt{\sigma_{r}^{2}+\sigma_{x}^{2}} \sqrt{\sigma_{r^{\prime}}^{2}+\sigma_{x^{\prime}}^{2}} \sqrt{\sigma_{r}^{2}+\sigma_{y}^{2}} \sqrt{\sigma_{r^{\prime}}^{2}+\sigma_{y^{\prime}}^{2}}},
$$

where $\sigma_{r}=\sqrt{\lambda} \bar{N} \lambda_{0} / 4 \pi, \sigma_{r^{\prime}}=\sqrt{\lambda / N} \lambda_{0}$, and the rest of the $\sigma$ 's are the transverse rms beam sizes and beam angular spreads at the undulator. Assuming a symmetrical $\beta$ function of $1 \mathrm{~m}$ at the middle of the undulator, and the emittance in Table I, the rms beam sizes at the undulator are $0.62 \mathrm{~mm}$ and the angular spreads are $620 \mu \mathrm{rad}$. Now $\sigma_{r}=1.5 \mathrm{~mm}$ and $\sigma_{r^{\prime}}=15 \mathrm{mrad}$ when $N=25$, and the brilliance is largely determined by the photon diffraction effects.

Even though the beam average current is only $100 \mu \mathrm{A}$, the average $\mathrm{THz}$ beam power is orders of magnitude 
beyond that available from other nonaccelerator sources, is comparable to the demonstrated recirculated linac driven $\mathrm{THz}$ source at Jefferson Lab which is physically much bigger, much more expensive, and requires a much higher average beam current and bunch charge [20], and is greater than the powers being talked about from storage rings [6].

In this device wavelength tuning may be accomplished in three different ways, two of which are well known from free electron lasers. Because the emission wavelength depends on the energy, covering the range $0.3-3 \mathrm{THz}$ is accomplished by changing the electron beam energy between 3.1 and $9.9 \mathrm{MeV}$. Such changes are relatively easy to accomplish by (i) scaling the magnetic fields in the recirculation system by the energy ratio, (ii) adjusting the rf cavity operating amplitude down by the correct amount, and (iii) adjusting the rf cavity phase slightly to achieve maximum bunch compression at the undulator. One may also change the wavelength by changing the magnetic field strength in the undulator. This method is probably preferred for small frequency changes, but becomes cumbersome if a large change of frequency is to be accomplished. Because one is operating at low energy in this device, it is also possible to change the photon emission wavelength observed by observing at an angle to the undulator axis due to Doppler shift of the emitted frequency. There will be some reduction of flux and brilliance by observing off the forward direction, but this scheme may be preferred in cases where the overhead of resetting the accelerator conditions needs to be avoided.

There has been much recent activity within the storage ring community about utilizing storage rings as $\mathrm{THz}$ radiation sources. Better high average power $\mathrm{THz}$ sources, especially ones that might be of cost and size accessible to a university department, could allow much more rapid exploration of still uncovered science in the $\mathrm{THz}$ band. Linacs and recirculated linacs will always have a large advantage compared to storage rings regarding the ultimate compression of bunches possible, because storage rings will always be limited by quantum excitation of synchrotron oscillations [21]. Small superconducting storage rings, indeed not optimized for the production of $\mathrm{THz}$, have been built and several hundred $\mathrm{MeV}$ beams can be stored in devices that fit in a large room. On the other hand, the device described in this paper, particularly in its low average current versions, has the potential to be a table top device.

In this paper a specific rendering of a compact $\mathrm{THz}$ source has been presented and, more importantly, a physical limit of the use of small recirculated linacs for producing $\mathrm{THz}$ has been explored. A main limit to bunch charge in such a source is the energy detuning due to the coherent emission of energy in the source itself. As this detuning goes down with the number of undulator periods and with the field strength, one tends towards undulators with smaller numbers of periods and smaller field strengths, consistent with one's ability to compress the bunches to short distances. This conclusion is in marked contrast to undulators designed for $\mathrm{x}$-ray production in storage rings. Here one enhances $\mathrm{x}$-ray flux and brilliance by building many periods with as short a period length as possible.

\section{ACKNOWLEDGMENTS}

This work supported by the U.S. Department of Energy under Contract No. DE-AC05-84ER40150.

[1] M. Abo-Bakr, J. Feikes, K. Holldack, G. Wüstefeld, and H.-W. Hübers, Phys. Rev. Lett. 88, 254801 (2002).

[2] J. M. Byrd, W. P. Leemans, A. Loftsdottir, B. Marcelis, M. C. Martin, W. R. McKinney, F. Sannibale, T. Scarvie, and C. Steier, Phys. Rev. Lett. 89, 224801 (2002).

[3] M. Venturini and R. Warnock, Phys. Rev. Lett. 89, 224802 (2002).

[4] G. Stupakov and S. Heifets, Phys. Rev. ST Accel. Beams 5, 054402 (2002).

[5] G. L. Carr, S. L. Kramer, J. B. Murphy, R. P. S. M. Lobo, and D. B. Tanner, Nucl. Instrum. Methods Phys. Res., Sect. A 463, 387 (2001).

[6] M. Abo-Bakr, J. Fiekes, K. Holldack, P. Kuske, W. B. Peatman, U. Schade, G. Wüstefeld, and H.-W. Hübers, Phys. Rev. Lett. 90, 094801 (2003).

[7] T. Nakazato, M. Oyamada, N. Niimura, S. Urasawa, O. Konno, A. Kagaya, R. Kato, T. Kamiyama, Y. Torizuka, T. Nanba, Y. Kondo, Y. Shibata, K. Ishi, T. Ohsaka, and M. Ikezawa, Phys. Rev. Lett. 63, 1245 (1989).

[8] U. Happek, A. J. Sievers, and E. B. Blum, Phys. Rev. Lett. 67, 2962 (1991).

[9] C. P. Neumann, W. S. Graves, and P. G. O'Shea, Phys. Rev. ST Accel. Beams 3, 030701 (2000).

[10] D.X. Wang, in Proceedings of the 1997 Particle Accelerator Conference, Vancouver, Canada, 1997 (IEEE, Piscataway, NJ, 1998), p. 1976.

[11] G. A. Krafft, in Proceedings of the Third European Workshop on Beam Diagnostics and Instrumentation in Particle Accelerators, Rome, 1997 (LNF-INFN, Frascati, 1997), p. 48.

[12] C.W. Leemann, D. R. Douglas, and G. A. Krafft, Annu. Rev. Nucl. Part. Sci. 51, 413 (2001).

[13] G. Kulypanov, A. Skrinsky, and N. Vinokurov, J. Synchrotron Radiat. 5, 176 (1998).

[14] Sol M. Gruner, Don Bilderback, Ivan Bazarov, Ken Finkelstein, Geoffrey Krafft, Lia Merminga, Hasan Padamsee, Qun Shen, Charles Sinclair, and Maury Tigner, Rev. Sci. Instrum. 73, 1402 (2002).

[15] G. A. Krafft, in Proceedings of the 1999 Particle Accelerator Conference, New York, 1999 (IEEE, Piscataway, NJ, 1999), p. 2448.

[16] D. X. Wang, G. A. Krafft, and C. K. Sinclair, Phys. Rev. E 57, 2283 (1998). 
[17] G. R. Neil, C. L. Bohn, S.V. Benson, G. Biallas, D. Douglas, H. F. Dylla, R. Evans, J. Fugitt, A. Grippo, J. Gubeli, R. Hill, K. Jordan, G. A. Krafft, R. Li, L. Merminga, P. Piot, J. Preble, M. Shinn, T. Siggins, R. Walker, and B. Yunn, Phys. Rev. Lett. 84, 662 (2000).

[18] L. D. Landau and E. M. Lifshitz, The Classical Theory of Fields (Pergammon, Oxford, 1975), 4th ed., p. 202, Eq. 74.14.
[19] K.-J. Kim, in Physics of Particle Accelerators, edited by Melvin Month and Margaret Dienes, AIP Conf. Proc. No. 184 (AIP, New York, 1989), p. 565.

[20] G. L. Carr, M. C. Martin, W. R. McKinney, K. Jordan, G. R. Neil, and G. P. Williams, Nature (London) 420, 153 (2002).

[21] G. A. Krafft, in Proceedings of the 2002 Joint Accelerator School, Long Beach, CA, 2002 (World Scientific, Singapore, 2004), p. 301. 